\title{
Utilização da Ferramenta Five Labs para Identificação de Traços de Personalidade dos Estudantes
}

\author{
Janderson J. B. Aguiar ${ }^{1}$, Joseana M. Fechine', Evandro B. Costa ${ }^{2}$ \\ ${ }^{1}$ Universidade Federal de Campina Grande (UFCG), Campina Grande - PB - Brasil \\ ${ }^{2}$ Universidade Federal de Alagoas (UFAL), Maceió - AL - Brasil \\ janderson@copin.ufcg.edu.br, joseana@dsc.ufcg.edu.br, evandro@ic.ufal.br
}

\begin{abstract}
Increasingly there is concern in dealing with students on an individual way. Personality is a peculiar aspect of every individual that can influence the process of teaching and learning. The purpose of this paper is to present an experience report, in the school space, involving the use of Five Labs, a tool to extract the Personality Traits of students based on their posts on Facebook. With Five Labs, it was possible to identify the profile of three classes of students of computer science at technical level. It is hoped that this paper encourage the identification of this psychological aspect in schools, and encourage more research on Personality in Computers in Education.
\end{abstract}

Resumo. Cada vez mais há preocupação em tratar os alunos de maneira individualizada. A Personalidade é um aspecto peculiar de cada indivíduo que pode influenciar no processo de ensino e aprendizagem. O objetivo deste artigo consiste em apresentar um relato de experiência, no espaço escolar, envolvendo o uso da ferramenta Five Labs para, com base em postagens na rede social Facebook, extrair os Traços de Personalidade dos alunos. Com esta ferramenta, foi possível identificar com facilidade o perfil de três turmas de alunos de informática em nível técnico. Espera-se com este artigo incentivar a identificação desse aspecto psicológico nas escolas, além de incentivar mais pesquisas sobre Personalidade em Informática na Educação.

\section{Introdução}

Segundo Litto (1996 apud Kuri 2004), deve-se haver uma educação individualizada, sendo inaceitável que se continue com o paradigma industrial de tratar todos os alunos iguais, como em uma linha de montagem. Apesar de ser um citação de quase 20 anos, permanece atual por ainda se perceber muitas vezes uma educação não individualizada nas escolas. Nesse sentido, é relevante utilizar, no contexto escolar, ferramentas que ajudem a tratar os alunos de maneira particularizada.

As tecnologias de informação e comunicação podem servir de mediadoras para considerar aspectos psicológicos humanos na educação. A personalidade, por exemplo, é um aspecto psicológico que influencia no processo de tomada de decisão [Nunes 2012] e, assim sendo, é importante considerá-la no processo de ensino e aprendizagem.

Apesar de a definição do conceito de personalidade não ser consensual, é possível defini-la como um padrão de comportamento consistente originado internamente no indivíduo [Burger 2000].

Neste artigo, é apresentado um relato de experiência de uso de uma ferramenta on-line (Five Labs) para identificar os traços de personalidade de alunos a partir de seus 
perfis na rede social Facebook (https://www.facebook.com/). Com base nisso, são também realizados comentários para estimular reproduções similares dessa experiência.

O restante do artigo está estruturado da seguinte forma: na seção 2, é apresentada a teoria de personalidade baseada em traços; na seção 3, são comentados trabalhos relacionados; na seção 4, é explanado como foi organizada a experiência de uso da ferramenta Five Labs; na seção 5, são discutidos os resultados obtidos; e, na seção 6, são apresentados comentários finais baseados nessa experiência realizada.

\section{A Teoria dos Traços de Personalidade}

Por ser mais fácil mensurar por meio computacional, a Teoria dos Traços [Allport e Allport 1921] é uma das teorias mais populares para representar o conceito de personalidade em pesquisas de áreas tecnológicas.

O modelo conhecido como Big Five [John e Srivastava 1999] consiste na versão moderna da Teoria dos Traços. Os cinco fatores deste modelo são: Extroversão (extraversion), Socialização (agreeableness), Realização (conscientiousness), Neuroticismo (neuroticism) e Abertura (openess). No Quadro 1, encontram-se considerações relativas a cada um desses fatores, com base nas pesquisas de Costa e Widiger (2002), Costa e McCrae (2007), Nunes, Hutz e Nunes (2010) e Rabelo (2013).

\section{Quadro 1. Características dos fatores do Big Five.}

Extroversão refere-se às formas como os indivíduos interagem com os outros, indicando o quanto são comunicativos, falantes, ativos, assertivos, responsivos e gregários. Índices altos tendem, portanto, a representar pessoas mais sociáveis, ativas, falantes, otimistas, alegres, bem dispostas e cordiais. Entretanto, índices baixos não representam necessariamente pessoas infelizes, não amistosas ou pessimistas, mas tendem a representar pessoas reservadas, introvertidas, sóbrias, indiferentes, independentes e quietas.

Socialização refere-se à qualidade das relações interpessoais dos indivíduos e aos tipos de interações que um indivíduo apresenta - predisposição para ser amistoso, para sensibilizar-se com a situação alheia, para colocar-se no lugar do outro, para demonstrar gentileza, complacência, compaixão. Índices altos tendem, portanto, a representar pessoas generosas, amáveis, afetuosas, prestativas e altruístas, com tendência à responsabilidade e empatia, e que acreditam que a maioria das outras pessoas agirá da mesma forma. Por outro lado, índices baixos tendem a representar pessoas petulantes, não cooperativas, facilmente irritáveis, e que podem apresentar-se de maneira manipuladora, vingativa e insensível.

Realização refere-se ao grau de organização e condução de tarefas, persistência, motivação, controle e autocontrole em termos de planejamento apresentados pelos indivíduos. Índices altos tendem, portanto, a representar pessoas mais organizadas, confiáveis, trabalhadoras, determinadas, pontuais, meticulosas, ambiciosas e persistentes. Índices baixos tendem a representar pessoas preguiçosas, descuidadas, desorganizadas, negligentes, que não têm objetivos claros, e que tendem a ter pouco comprometimento e responsabilidade diante de tarefas.

Neuroticismo refere-se à tendência cotidiana dos indivíduos a experimentar padrões emocionais vinculados a desconforto psicológico (aflição, angústia, culpa, medo, nojo, raiva, sofrimento, tristeza, vergonha, entre outros), além de referir-se à ansiedade excessiva, à pouca habilidade para conter impulsos e para lidar com o estresse, à propensão a ideias dissociadas da realidade (irracionais), e à dificuldade para tolerar a frustração causada pela não realização de desejos. Índices altos tendem, portanto, a representar pessoas predispostas a vivenciar mais profundamente o seu sofrimento emocional. Por outro lado, índices baixos tendem a representar pessoas mais tranquilas, relaxadas, constantes, menos agitadas, com habilidade maior em lidar com situações estressantes e, em síntese, com estabilidade emocional.

Abertura refere-se aos comportamentos exploratórios, ao reconhecimento da importância de ter novas experiências, à imaginação ativa, à sensibilidade estética, à curiosidade intelectual e à preferência pela variedade. Índices altos tendem, portanto, a representar pessoas curiosas, criativas, imaginativas, que se divertem com novas ideias, que costumam apresentar valores não convencionais, que experienciam intensamente uma gama ampla de emoções. Por outro lado, índices baixos tendem a representar pessoas convencionais, dogmáticas, rígidas nas suas crenças e atitudes, conservadoras nas suas preferências e menos responsivas emocionalmente. 
Foram criados vários inventários para realizar pesquisas com base no Big Five, sendo alguns dos mais utilizados o NEO-PI-R (Revised NEO Personality Inventory), com 240 questões de múltipla escolha [Costa e Mccrae 1992], e o NEO-IPIP (NEO International Personality Item Pool) com 300 itens [Johnson 2000].

Apesar do uso comum de inventários, há estudos considerando abordagens que não exigem muito esforço cognitivo por parte dos usuários para detectar seus traços de personalidade. Mairesse et al. (2007) e Tausczik e Pennebaker (2009), mostram que há correlação entre o Big Five e características linguísticas encontradas em textos. Bachrach et al. (2012), Markovikj et al. (2013) e Schwartz et al. (2013) apresentaram estudos indicando que Traços de Personalidade possuem correlação com padrões de uso da rede social Facebook, podendo facilitar a detecção de personalidade com a mineração dos dados dos perfis de usuário dessa rede social.

$\mathrm{Na}$ pesquisa de Schwartz et al. (2013), por exemplo, foram analisados 700 milhões de palavras, frases e tópicos coletados de 75 mil voluntários usuários do Facebook, que também responderam ao NEO-IPIP. A acurácia foi de 91,9\%. Com base nesse estudo, foi desenvolvida uma ferramenta para análise semântica das postagens do Facebook, Five Labs, disponibilizada pela startup americana Five [Five 2015].

\section{Pesquisas Relacionadas}

Muitos trabalhos analisando a personalidade de indivíduos podem ser encontrados na literatura, em diversas domínios, tais como Capretz e Ahmed (2010) e Cruz et al. (2011) - cujo enfoque consistiu em considerar o conceito de personalidade em tarefas relativas à Engenharia de Software -, e Rabelo (2013) - que investigou os traços de personalidade em atletas brasileiros.

No contexto educacional, há pesquisas como a de Paixão, Fortaleza e Conte (2012, 2013), que realizaram estudos sobre a personalidade no ensino de computação, discutindo implicações dos perfis psicológicos a partir de uma análise inicial com 46 alunos de Ciência da Computação e Sistemas de Informação, e posteriormente com 80 alunos, relacionando também o perfil psicológico com a evasão.

Nessa mesma linha, Farias, Dobrões e Da Silva (2013) realizaram uma análise para adaptar atividades da área de computação considerando os perfis psicológicos dos alunos, para ajudar na aprendizagem e no desempenho acadêmico. Para o ensino de engenharia, Kuri (2004) estudou a personalidade dos alunos de graduação em Engenharia Civil, Mecânica Elétrica e Produção.

Apesar dos trabalhos citados nesta seção - além de alguns comentados na seção 2 - estarem relacionados ao trabalho descrito neste artigo, eles diferenciam-se deste por não utilizarem uma ferramenta que, com base nos perfis na rede social Facebook, extraiam sua personalidade objetivando propiciar melhorias no âmbito educacional. Além disso, não foram encontrados trabalhos com foco na Teoria dos Traços para detectar, sem uso de inventários, os traços de personalidade de alunos de cursos técnicos em informática - e isto consiste na experiência relatada neste artigo.

É interessante mencionar que, ao usar os termos de busca personalid* e personalit* (visando explorar todos os trabalhos em português, inglês ou espanhol que citassem algo sobre personalidade) nos engenhos de busca dos anais do SBIE (Simpósio Brasileiro de Informática na Educação), WIE (Workshop de Informática na Escola), e 
do periódico RBIE (Revista Brasileira de Informática na Educação) - que são os veículos brasileiros melhor avaliados pela CAPES na área de Informática na Educação - nenhum dos poucos trabalhos retornados (apenas 8) apresentaram o foco descrito no parágrafo anterior. Além disso, é importante destacar a não ocorrência de pesquisas resultantes na busca dos anais do WIE. Isso indicia a escassez de estudos utilizando o conceito de Personalidade em Informática na Educação.

\section{Metodologia}

Com base na Teoria dos Traços, explanada na seção 2, foi identificada a personalidade de alunos de 3 (três) turmas do Curso Técnico em Informática da Escola Técnica Redentorista (Campina Grande - PB). Uma vez que essas turmas tinham tamanho variado, a quantidade de resultados variou por turma, sendo 25 para uma das turmas (denominada neste artigo por Turma A), 7 para outra turma (denominada neste artigo por Turma B), e 17 para uma terceira turma (denominada neste artigo por Turma C). Ao todo, portanto, foram 49 alunos com os traços de personalidade extraídos via Five Labs.

A ferramenta Five Labs analisa a linguagem que os usuários do Facebook utilizam nesta rede social. O modelo é baseado no estudo de Schwartz et al. (2013), examinando o conteúdo linguístico das postagens no Facebook, usando palavras-chave para realizar estimativas precisas sobre a personalidade dos indivíduos [Five 2015].

Os resultados de cada aluno foram obtidos para análise por meio de um formulário criado via Google Drive (https://drive.google.com/), cujo hiperlink foi enviado para o e-mail dos alunos. Em tal formulário, havia informações gerais, em português, sobre a ferramenta (uma vez que ela está disponível em inglês), além de cinco campos para cada aluno preencher a porcentagem apresentada na ferramenta relativa a cada um dos traços do Big Five.

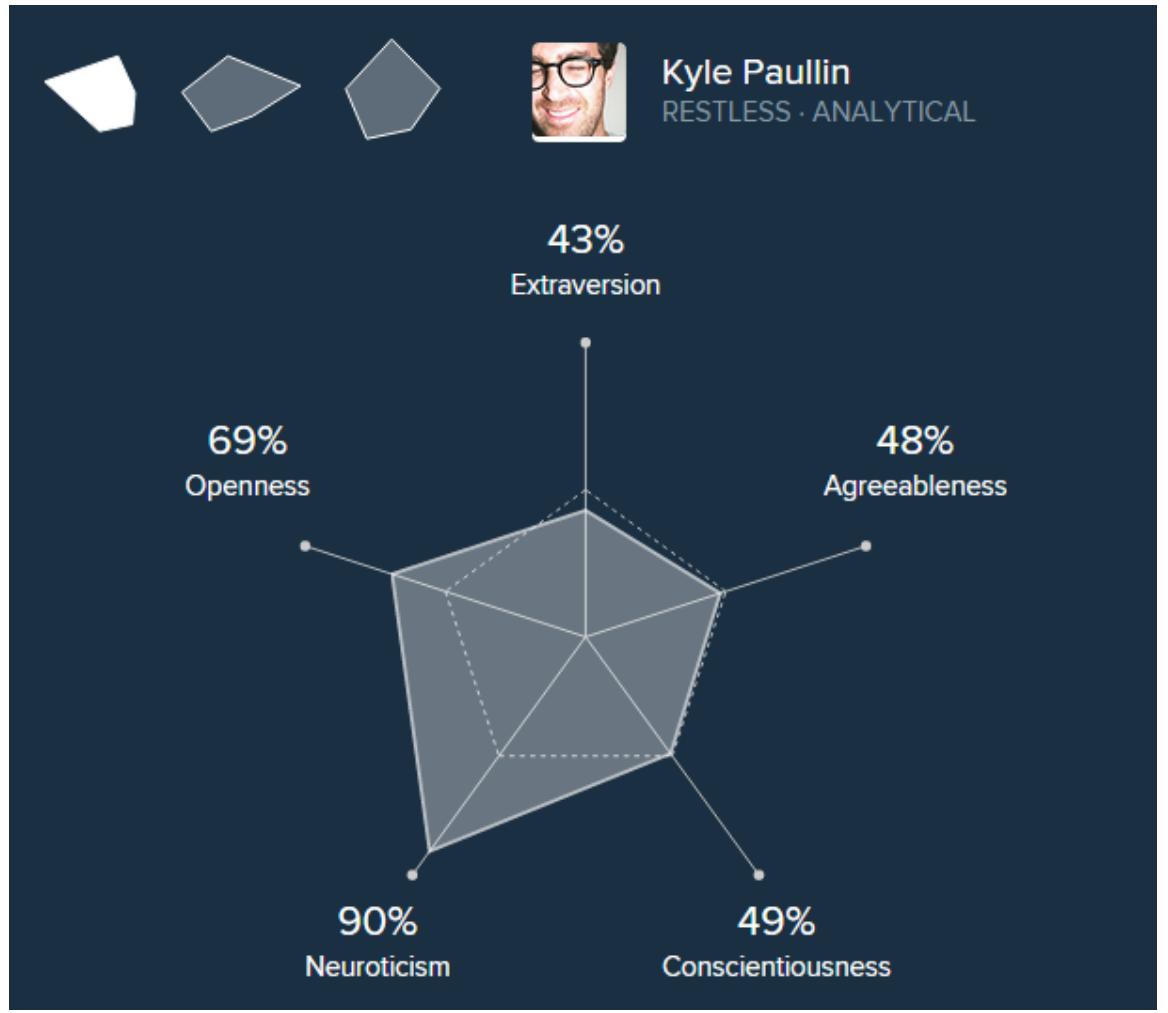

Figura 1. Ilustração da ferramenta Five Labs. Fonte: Extraído de [Five 2015]. 
Considerando os valores percentuais providos pela Five Labs (como ilustrado na Figura 1), foi definido que: (i) para 0 a 35\%, o indivíduo possui um nível baixo para aquele traço; (ii) de 65 a 100\%, o indivíduo possui um nível alto para aquele traço; e (iii) entre 35 a $65 \%$, considerou-se que tal traço é encontrado moderadamente no indivíduo. Por exemplo, para um aluno cujo resultado disponibilizado foi $15 \%, 30 \%$, $80 \%, 45 \%$ e $55 \%$, respectivamente para os traços Extroversão, Socialização, Realização, Neuroticismo e Abertura, é possível afirmar que ele se apresenta como um indivíduo com Extroversão e Socialização baixas, Neuroticismo alto, e Realização e Abertura moderadas.

\section{Análise de Resultados}

A partir da metodologia apresentada na seção 4, foram elaborados os gráficos do Quadro 2 para ilustrar os resultados obtidos.

Com base no Quadro 2, na linha relativa ao Traço Extroversão, percebe-se que a maioria apresenta um nível moderado de Extroversão, mas a quantidade de alunos com Extroversão alta é significativa. É interessante verificar também que, na Turma $C$, diferentemente das demais, há uma porcentagem de alunos, com nível baixo de Extroversão, que deve ser levada em consideração. Ou seja, na Turma A e na Turma B, poderiam ser adotadas estratégias mais voltadas a indivíduos extrovertidos, enquanto que, na Turma $\mathrm{C}$, se deve ter mais cuidado por conter uma parcela considerável de alunos com tendência introvertida de comportamento.

Com base na linha do Quadro 2 relativa ao Traço Socialização, nota-se que, em todas as turmas, a maioria foi considerada com Socialização baixa, não havendo casos de Socialização Alta. A partir dessa análise, o docente poderia considerar a turma de forma mais homogênea, mas é importante destacar que há alguns com o nível moderado, especialmente na Turma C, devendo-se ter cuidado ao propor apenas atividades que privilegiariam o perfil de Socialização baixa.

Similarmente ao traço Socialização, pelos gráficos do Quadro 2 relativos ao Traço Realização, é indicado que a maioria dos alunos apresentam um nível baixo, não havendo casos de nível alto de Realização.

Considerando a linha do Quadro 2 relativa ao Traço Neuroticismo, percebe-se que a maioria dos alunos apresenta um nível moderado de Neuroticismo. Entretanto, ao analisar por turma, visivelmente percebe-se que é interessante os docentes terem um cuidado com alguns alunos, uma vez que em cada turma há ocorrências de alunos com Neuroticismo alto, ou seja, alta instabilidade emocional.

Com base na linha do Quadro 2 relativa ao Traço Abertura, é ilustrado que os alunos apresentaram em geral um perfil com Abertura alta, ou pelo menos moderada. Especialmente na Turma A, acredita-se que o docente poderia mudar suas estratégias metodológicas durante o curso, e a turma estaria aberta a essas mudanças. Entretanto, na Turma B e na Turma C, inovações metodológicas por parte do professor provavelmente não seriam tão bem aceitas por alunos com Abertura moderada, até possivelmente prejudicando o aprendizado.

É importante reforçar o fato de que se deve verificar os traços de personalidade tanto por turma quanto individualmente, para que o professor busque estratégias mais satisfatórias e personalizadas ao propor atividades aos alunos. 
CBIE-LACLO 2015

Anais do XXI Workshop de Informática na Escola (WIE 2015)

Quadro 2. Resultados da utilização do Five Labs em três turmas de alunos.

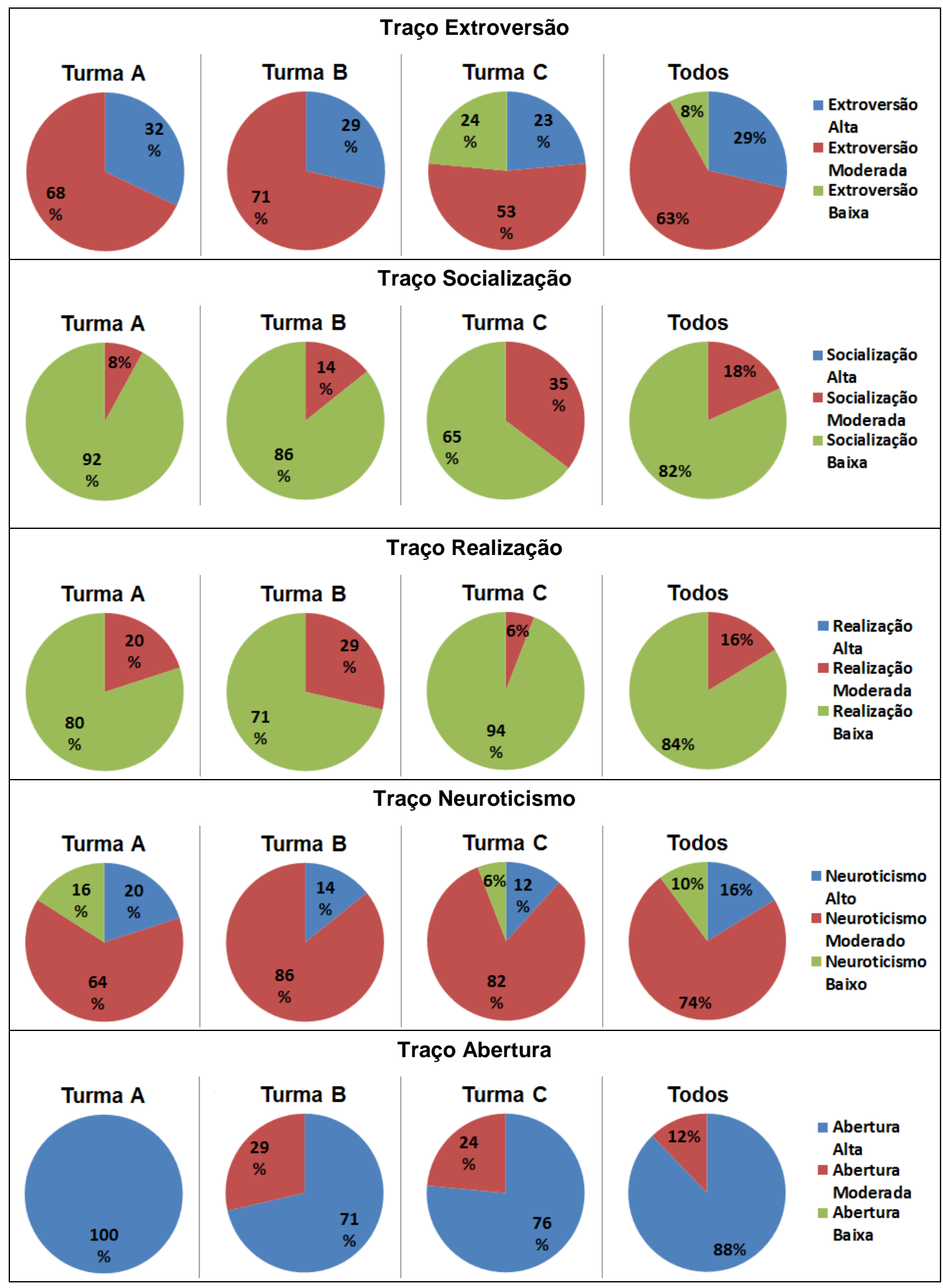

Além dos gráficos do Quadro 2, foi analisado por turma o nível de similaridade entre as personalidades dos alunos, para identificar os perfis mais diferentes, considerando os cinco traços de personalidade. Para isso, os cinco valores percentuais formaram um vetor de usuário e, uma vez que cada usuário estava sendo representado 
por um vetor, foi utilizada a medida de Similaridade dos Cossenos para comparar esses vetores. No Quadro 3 são apresentados gráficos do tipo radar que ilustram os perfis de alunos mais diferentes, por turma, considerando tal medida de similaridade.

Quadro 3. Comparação, por turma, de perfis distintos de personalidade de alunos.

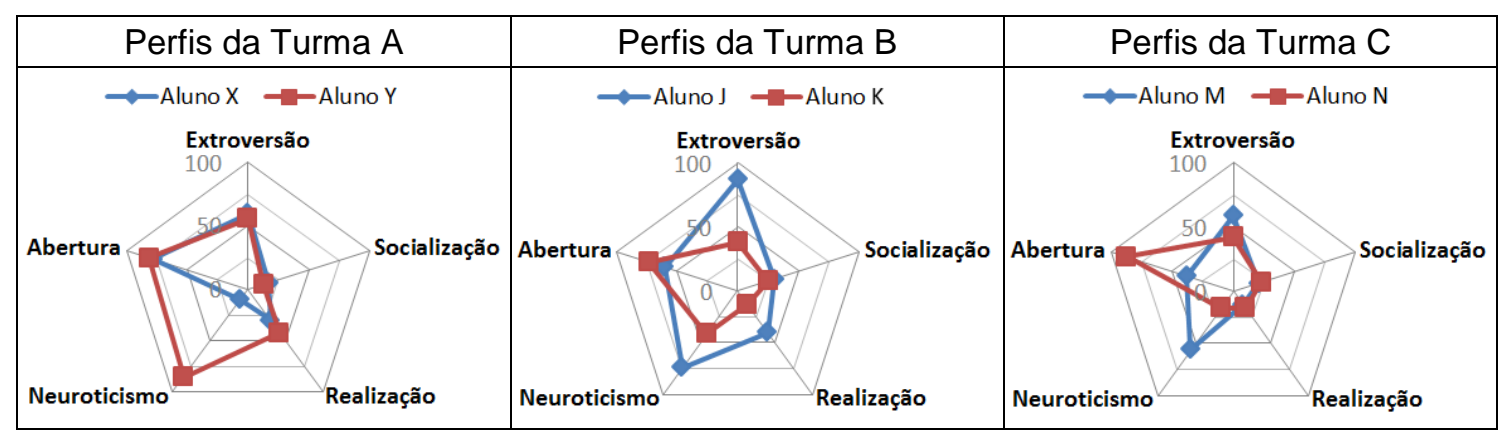

Com base nos gráficos do Quadro 3, é possível observar que os alunos do curso técnico em informática de uma mesma turma, que participaram desta experiência, podem apresentar algumas características bem diferentes, a exemplo do traço Neuroticismo no gráfico que ilustra os perfis da Turma $A$, do traço Extroversão no gráfico que ilustra os perfis da Turma $\mathrm{B}$, e do traço Abertura no gráfico que ilustra os perfis da Turma C.

Apesar das diferenças, percebeu-se nesta experiência que, em muitos casos, os alunos são similares, como sugerem alguns traços nos gráficos do Quadro 3, além dos gráficos do Quadro 2. A partir do Quadro 4, é possível observar que há perfis de alunos do curso técnico em informática com personalidade quase $100 \%$ similares. A menor similaridade observada, considerando todos os alunos, foi de aproximadamente $76 \%$, sendo de $78 \%$ a menor similaridade observada por turma.

\section{Quadro 4. Similaridades entre alunos de um Curso Técnico em Informática.}

\begin{tabular}{|l|c|c|c|c|}
\hline & Turma A & Turma B & Turma C & Todos \\
\hline Maior Similaridade & 0,99965 & 0,99388 & 0,99798 & 0,99982 \\
\hline Menor Similaridade & 0,83489 & 0,90109 & 0,77717 & 0,75566 \\
\hline Similaridade Média & 0,96633 & 0,96288 & 0,93473 & 0,95452 \\
\hline
\end{tabular}

Com a realização desta experiência, foi possível perceber que, apesar de haver bastante similaridade no perfil dos alunos em um curso técnico em informática, relativo à sua personalidade, é possível encontrar alunos com características peculiares e que, se forem utilizadas estratégias sempre considerando a homogeneidade da turma, estes alunos tendem a ser prejudicados.

A análise individual dos traços de personalidade e, por conseguinte, uma visão geral de todos os traços encontrados nas turmas, podem ajudar a prever que alguns aspectos metodológicos a serem adotados podem ser mais úteis em relação aos outros.

Indivíduos, por exemplo, caracterizados com pouca Extroversão e Socialização, podem obter resultados satisfatórios em trabalhos individuais e/ou provas escritas; por outro lado, podem obter resultados insatisfatórios em apresentações de seminários e/ou trabalhos em equipes. Embora haja casos em que a maioria da turma apresente perfis similares, é papel dos docentes propiciar um aprendizado adequado a todos os alunos, mesmo que seja mais trabalhoso realizar adaptações para os mais diferenciados. 
As três turmas do curso técnico em informática, de modo geral, apresentaram-se similares, mas é importante reforçar que analisar as características individuais dos alunos é muitas vezes interessante e necessário, principalmente visualizando os valores percentuais sem agrupá-los em intervalos. Com base nisso, o gráfico da Figura 2, com os cinco traços combinados por indivíduo, ilustra que os alunos apresentam-se diferentes. No eixo horizontal, têm-se a variação para os 49 estudantes. No eixo vertical, os valores indicam a porcentagem (de $0 \%$ a $100 \%$ ) para cada traço, representado por símbolos/cores diferentes. Apesar de nesta experiência os percentuais não variarem com maior intensidade (por exemplo, os valores para Socialização e Realização são em geral baixos), são visíveis as variações em todo o eixo horizontal, indiciando e reforçando que cada aluno tem suas particularidades.

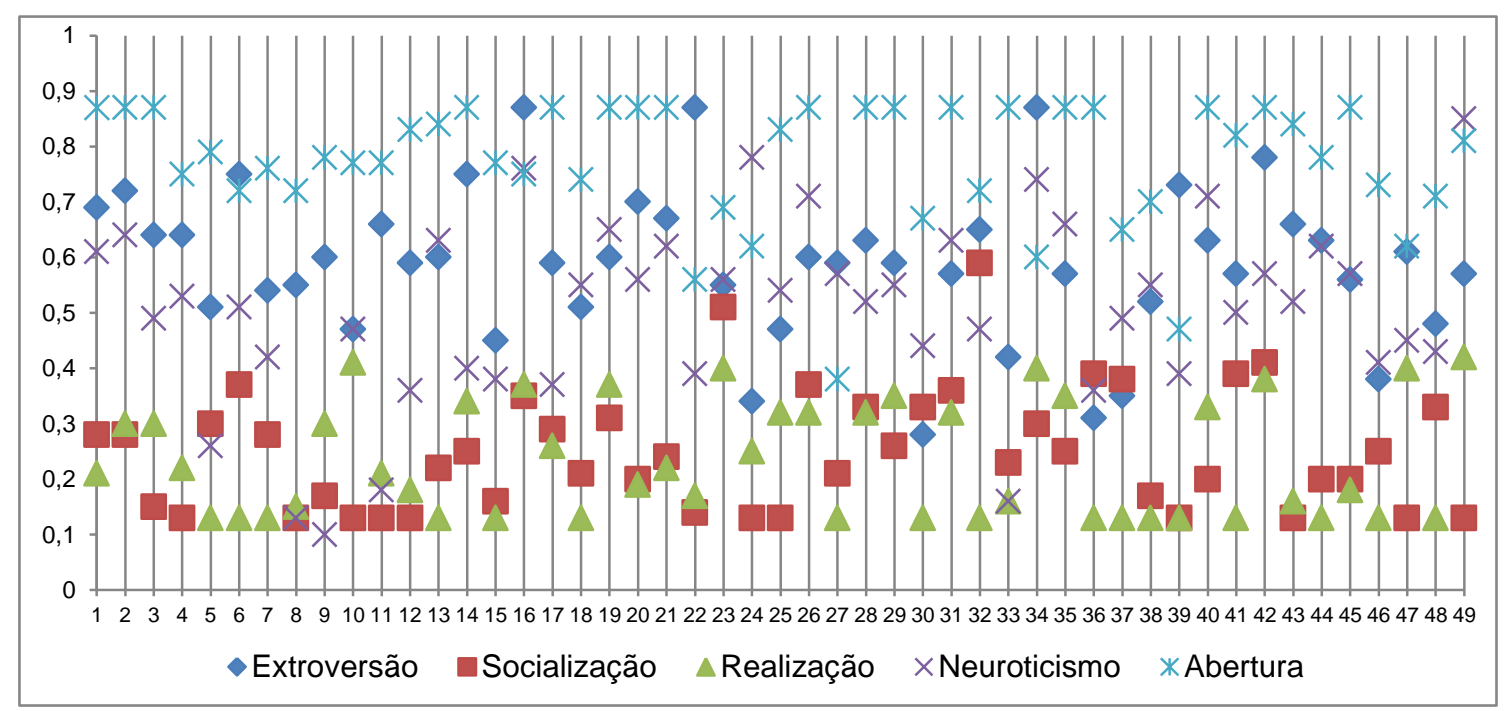

Figura 2. llustração dos dados percentuais para cada fator do Big Five relativos a cada um dos 49 estudantes participantes desta pesquisa.

\section{Considerações Finais}

A realização da experiência relatada neste artigo propiciou a visualização de perfis de alunos de três turmas em relação à personalidade, baseando-se na ferramenta Five Labs. A interação humana se faz necessária em diversas atividades. A personalidade de cada individuo influencia na interação com outros indivíduos e, portanto, pode impactar na realização de atividades no contexto escolar.

Uma vez que a personalidade influencia os relacionamentos aluno-professor e aluno-aluno, há consequentemente influência em aspectos educacionais como desempenho e evasão. Além disso, alguns pesquisadores, a exemplo de Kuri (2004), Zonash e Naqvi (2011) e Raju e Venugopal (2014), mostram resultados a respeito de correlações entre personalidade e teorias sobre os estilos de aprendizagem preferidos por cada aluno.

É importante que os docentes (e até mesmo instâncias mais abrangentes, como coordenadores de curso e direção escolar) reflitam a possibilidade de extrairem os traços de personalidade dos alunos de suas turmas, no início das disciplinas, o que pode ser algo sistematicamente planejado e compartilhado com todos os docentes.

Ao identificarem determinados traços de personalidade a partir do uso de ferramentas (como o Five Labs e/ou outros meios, uma vez que há alunos que não usam 
redes sociais), os professores poderiam indicar às coordenações ou outros responsáveis da escola a necessidade de atenção a determinados alunos, não objetivando discriminação, mas o cuidado, sugerindo em alguns casos aos pais/responsáveis o acompanhamento de alunos por psicólogos.

Além disso, as escolas que têm a presença de psicólogos em seu quadro de funcionários poderiam atribuir a estes a responsabilidade pelo diagnóstico e acompanhamento mais apropriado, verificando melhor os resultados prévios (obtidos pelos docentes por instrumentos de mensuração como o Five Labs), e analisando o impacto no aprendizado, no convívio social, e mesmo na formação cidadã, o que também é função da escola.

Além de pertinentes no contexto de como o aluno se relaciona com seus colegas e professores em sala de aula, os conceitos sobre os traços de personalidade podem ser empregados, por exemplo, na personalização de ambientes virtuais de ensino e aprendizagem, indispensáveis para cursos a distância e também empregados como apoio em cursos presenciais.

Por fim, mesmo os que desconheciam a Teoria do Big Five antes da leitura deste artigo, acredita-se que, neste ponto, com base nas características relativas a cada um dos traços comentadas na seção 2, é possível compreender a importância de detectar tais características dos alunos no espaço escolar, facilitando o convívio aluno-aluno e aluno-professor.

Almeja-se com este artigo motivar mais pesquisas sobre o uso de informações relativas à personalidade dos alunos no contexto educacional, realizando a detecção por meio da mineração de dados de perfis de usuários em redes sociais (como apresentado neste artigo) ou outros meios, como a aplicação dos tradicionais inventários de personalidade, que também podem ser aplicados com apoio das tecnologias de informação e comunicação.

\section{Agradecimentos}

À Escola Técnica Redentorista de Campina Grande - PB, especialmente aos alunos que participaram desta pesquisa.

\section{Referências}

Allport, F. H.; Allport, G. W. (1921). Personality Traits: Their Classification And Measurement. Journal Of Abnormal And Social Psychology, 16, p. 6-40.

Bachrach, Y.; Kosinski, M.; Graepel, T.; Kohli, P.; Stillwell, D. (2012). Personality and patterns of Facebook usage. In: Proceedings of the 3rd Annual ACM Web Science Conference (WebSci '12), ACM, New York, NY, USA, p. 24-32.

Burger, J. M. (2000). Personality. Wadsworth, fifth edition.

Capretz, L. F.; Ahmed, F. (2010). Why Do We Need Personality Diversity in Software Engineering?. ACM SIGSOFT Software Engineering Notes, v. 35, n. 2, p. 1-11.

Costa, P. T. Jr. ; Widiger, T. A. (2002). Introduction. In: Costa, P. T. Jr. ; Widiger, T. A (Org.), Personality Disorders and the Five-Factor Model of Personality, 2.ed., p.3-16. Whashington, DC: American Psychological Association.

Costa, P. T. Jr.; Mccrae, R. R. (2007). NEO PI-R: Inventário de Personalidade NEO Revisado e Inventário de Cinco Fatores NEO Revisado NEO-FFI-R [versão curta]. São Paulo: Vetor Editora Psico-Pedagógica. 
Costa, P. T.; Mccrae, R. R. (1992). Revised neo personality inventory (neo-pi-r) and neo five-factor inventory (neo-ffi). Professional manual.

Cruz, S.; Da Silva, F.; Monteiro, C.; Santos, P.; Rossilei, I. (2011). Personality in Software Engineering: preliminar findings from a systematic literature review. In: Proceedings of 15th Annual Conference on Evaluation \& Assessment in Software Engineering (EASE 2011), p. 1-10.

Farias, A. B.; Dobrões, J. A. L; Da Silva, R. Y. F. (2013). Strategies for Teaching Based on Academic Personality Types. In: XVIII Conferência Internacional sobre Informática na Educação (TISE), Porto Alegre-RS, p. 633-636.

Five. (2015). Five Labs - See the personality behind your posts. Product of Five.com. Disponível em: $<$ http://labs.five.com/>. Acesso em: 24 abr. 2015.

John, O. P.; Srivastava, S. (1999). The Big Five Trait taxonomy: History, measurement, and theoretical perspectives. In: Pervin, L. A.; John, O. P. (Org.), Handbook of Personality: Theory and research, v. 2, p. 102-138. Guilford Press, New York.

Johnson, J. A. (2000). Web-based personality assessment. In 71st Annual Meeting of the Eastern Psychological Association, USA.

Kuri, N. P. (2004). Tipos de personalidade e estilos de aprendizagem: proposições para o ensino de engenharia. 2004. Tese (Doutorado em Engenharia de Produção), Universidade Federal de São Carlos, São Carlos.

Mairesse, F.; Walker, M. A.; Mehl, M.; Moore, R. (2007). Using Linguistic Cues for the Automatic Recognition of Personality in Conversation and Text. Journal of Artificial Intelligence Research, p. 457-500.

Markovikj, D.; Gievska, S.; Kosinski, M.; Stillwell, D. (2013). Mining Facebook Data for Predictive Personality Modeling. Proc of Workshop on Computational Personality Recognition, AAAI Press, Melon Park, CA.

Nunes, C. H. S. S.; Hutz, C. S.; Nunes, M. F. O. (2010). Bateria Fatorial de Personalidade. Manual Técnico. São Paulo: Casa do Psicólogo.

Nunes, M. A. S. N. (2012). Computação Afetiva personalizando interfaces, interações e recomendações de produtos, serviços e pessoas em Ambientes computacionais. In: Nunes, M. A. S. N.; Oliveira, A. A.; Ordonez, E. D. M. (Org.). Projetos e Pesquisas em Ciência da Computação no DCOMP/PROCC/UFS: São Cristóvão, p. 115-151.

Paixão, C. C.; Fortaleza, L. L.; Conte, T. (2012). Um Estudo Preliminar sobre as Implicações de Tipos de Personalidade no Ensino de Computação. In: Anais do XXXII Congresso da Sociedade Brasileira de Computação (CSBC) - XX Workshop sobre Educação em Informática (WEI), Curitiba-PR.

Paixão, C. C.; Fortaleza, L. L.; Conte, T. (2013). Desafios no Ensino de Computação: um estudo da relação entre perfil psicológico de alunos e evasão. In: Anais do XXXIII Congresso da Sociedade Brasileira de Computação (CSBC) - XXI Workshop sobre Educação em Informática (WEI), MaceióAL, p. 720-729.

Rabelo, I. S. (2013). Investigação de traços de personalidade em atletas brasileiros: análise da adequação de uma ferramenta de avaliação psicológica. 2013. Tese (Doutorado em Pedagogia do Movimento Humano) - Escola de Educação Física e Esporte, Universidade de São Paulo, São Paulo.

Raju, P. G.; Venugopal, M. (2014). Personality \& learning styles — lessons for Indian corporate trainers. Indian Journal of Industrial Relations, 49(4).

Schwartz, H. A.; Eichstaedt, J. C.; Kern, M. L.; Dziurzynski, L.; Ramones, S. M.; Agrawal, M.; Shah, A.; Kosinski, M.; Stillwell, D.; Seligman, M. E. P.; Ungar, L. H. (2013). Personality, Gender, and Age in the Language of Social Media: The Open-Vocabulary Approach. PLoS ONE, 8(9), e73791.

Tausczik, Y. R.; Pennebaker, J. W. (2009). The Psychological Meaning of Words: LIWC and Computerized Text Analysis Methods. Journal of Language and Social Psychology, 29(1), p.24-54.

Zonash, R.; Naqvi, I. (2011). Personality Traits and Learning Styles among Students of Mathematics, Architecture, and Fine Arts. Journal of Behavioral scence, v. 21, p. 92-108. 